\title{
Weathering Server System Operations
}

\author{
D. Pawar ${ }^{1 *}$, S. C. Malik² and Reetu² \\ 'Amity Institute of Telecom Engineering and Management, Amity University, \\ Noida-201303, India; dpanwar75@yahoo.com \\ 2Department of Statistics, Maharshi Dayanand University, \\ Rohtak-124001, India; sc_malik@rediffmail.com
}

\begin{abstract}
In this paper reliability measures of a single-unit system operating under two weather conditions - normal and abnormal are evaluated using regenerative point technique. For this purpose, a reliability model is developed in which partial failure unit, fails totally. A single server randomly appears at partial failure of the unit on condition to visit immediately on complete failure of the system. The inspection of the unit at its partial failure is to assess the possibility of on-line repair. If not possible, it is repaired in down state. The repair of the unit at its complete failure is done without inspection. Server remains with the system in abnormal weather. The failure time of the unit and time of change of weather conditions are distributed exponentially; while the distributions of inspection and repair times are taken as arbitrary. Graphs are drawn to depict the behavior of some reliability and economic measures.
\end{abstract}

Keywords: Weather Conditions, Conditional Random Appearance of the Server, Inspection, On-line Repair, Partially Failure Stage (PFS), Reliability Measures.

\section{Introduction}

The life of a person has been made easier to a significant level by the rapidly growing technology. Everyone desires the latest technology in day-to-day life, wherein limited resources, restricts its usage. Therefore, preference is for more reliable and affordable single-unit systems. This encouraged many researchers including Dhillon and Natesan [1], Malik and Barak [2], Pawar et al. [3], Malik et al. [4], Malik and Pawar [5] for evaluating reliability of single-unit systems working under different weather conditions. Pawar and Malik [6] studied a single-unit system working under different weather conditions in which server visits the system immediately at its partial failure stage. But it is difficult for the server immediate attendance at any stage of failure. In such situations the server may precondition immediate attendance for the serious faults. Under this observation a reliability model is developed for a single-unit system operating under two weather conditions - normal and abnormal.

The system fails completely via partial failure. A single server is allowed to appear randomly at partial failure stage (PFS) subject to the condition that visit is made immediately at its complete failure. The inspection of the partially failed unit is carried out to see the possibility of on-line repair. If on-line repair is not possible, it is repaired in down state. However, repair of the unit at its complete failure is done without previous inspection. The inspection as well as repair activities are not allowed in abnormal weather. Server remains with the system in abnormal weather, while partially failed unit remains operative.

The failure time of the unit and time to change of weather conditions are distributed exponentially; while the distributions of inspection and repair times are taken as arbitrary. The random variables are considered as independent and uncorrelated. The control devices and repairs are

*Corresponding author:

D. Pawar (dpanwar75@yahoo.com) 
faultless. The expressions for various reliability measures such as mean sojourn times, mean time to system failure (MTSF), availability, busy period of the server, expected number of visits by the server and the profit function are derived using regenerative point technique. The graphical behavior of MTSF and Profit function with respect to abnormal weather rate has also been observed giving particular values to the parameters and costs.

\section{Notation}

$\mathrm{N}_{0} \quad$ : $\quad$ Unit is operative in normal mode

NA/A : Server is not available/ available

$\mathrm{PWi} / \mathrm{PUi}$ : Unit is partially failed and waiting for inspection/ under on-line inspection

PUr/PUrd: Unit is partially failed and under on-line repair/ under repair in down state

PWr/PWrd: Unit is partially failed and operative but waiting for repair/ waiting for repair in down state due to abnormal weather

FUr /FWr: Unit is completely failed and under repair in normal weather/ waiting for repair due to abnormal weather

$\mathrm{x} \quad$ : Constant rate of random appearance of the server

$r_{1} / r_{2}:$ Constant failure rate of the unit from normal to partial failure mode/ complete failure mode

$g(t) / g_{1}(t)$ : Repair rate of the unit from complete failure to the normal operative mode/ partial failure to the normal operative mode

$\beta / \beta_{1} \quad$ : Constant rate of change of weather from normal to abnormal/ abnormal to normal

$a h(t) / b h(t)$ : Rate of change of partially failed unit under inspection to on-line repair/ repair in down state

$\mathrm{q}_{\mathrm{ij}}(\mathrm{t}) / \mathrm{Q}_{\mathrm{jj}}(\mathrm{t})$ : Probability density function (pdf) and cumulative distribution function (cdf) of first passage time from regenerative state $i$ to a regenerative state $\mathrm{j}$ or to a failed state $\mathrm{j}$ without visiting any other regenerative state in $(0, t]$

$\mathrm{m}_{\mathrm{ij}} \quad$ : The unconditional mean time taken by the system to transit from any regenerative state $S_{j}$ when it (time) is counted from epoch of entrance in to that state $S_{j}$. Mathematically, it can be written as

$$
\mathrm{m}_{\mathrm{ij}}=\int_{0}^{\infty} \mathrm{td}\left[\mathrm{Q}_{\mathrm{ij}}(\mathrm{t})\right]=-\mathrm{q}_{\mathrm{ij}}^{*^{\prime}}(0)
$$

$\mu_{\mathrm{j}} \quad$ : Mean sojourn timeinstate $S_{\mathrm{j}}$ whichisgiven by $\mu_{\mathrm{j}}=\mathrm{E}(\mathrm{T})=\int_{0}^{\infty} \mathrm{P}(\mathrm{T}>t) \mathrm{dt}=\Sigma_{\mathrm{j}} \mathrm{m}_{\mathrm{ij}}$, where, $\mathrm{T}$ denotes the time to system failure

$\mathrm{M}_{\mathrm{j}}(\mathrm{t})$ : $\quad$ Probability that the system initially up in the regenerative state $S_{j}$ is up at time $t$ without passing through any other regenerative state

$W_{j}(t)$ : Probability that the server is busy at an instant $t$, given that the system entered into the regenerative state $S_{j}$ at $t=0$

$* * / * \quad:$ Symbol of Laplace Stieltjes Transform (LST)/ Laplace Transform (LT)

(S) $/ \mathrm{C} /{ }^{\prime}$ : Symbol of Stieltjes Convolution/ Laplace Convolution/ derivative of the function.

The regenerative transition states with transition rate for the system model are shown in Figure 1.

\section{Transition Probabilities and Mean Sojourn Times}

Simple probabilistic considerations yield the following expressions for the non-zero elements

$$
\mathrm{p}_{\mathrm{ij}}(\mathrm{t})=\mathrm{Q}_{\mathrm{ij}}(\infty)=\int \mathrm{q}_{\mathrm{ij}}(\mathrm{t}) \mathrm{dt} \quad \text { as }
$$

The transition probabilities are

$$
\begin{aligned}
& \mathrm{p}_{01}=1=\mathrm{p}_{98}, \mathrm{p}_{12}=\frac{\mathrm{x}}{\mathrm{x}+\mathrm{r}_{2}}, \mathrm{p}_{18}=\frac{\mathrm{r}_{2}}{\mathrm{x}+\mathrm{r}_{2}}, \mathrm{p}_{23}=\mathrm{ah}^{*}(\beta+\mathrm{r} 2), \\
& \mathrm{p}_{24}=\mathrm{bh}^{*}\left(\beta+\mathrm{r}_{2}\right), \mathrm{p}_{25}=\frac{\beta\left[1-\mathrm{h}^{*}\left(\beta+\mathrm{r}_{2}\right)\right]}{\beta+\mathrm{r}_{2}}, \mathrm{p}_{28}=\frac{\mathrm{r}_{2}\left[1-\mathrm{h}^{*}\left(\beta+\mathrm{r}_{2}\right)\right]}{\beta+\mathrm{r}_{2}}, \\
& \mathrm{p}_{30}=\mathrm{g}_{1}^{*}\left(\beta+\mathrm{r}_{2}\right), \mathrm{p}_{37}=\frac{\beta\left[1-\mathrm{g}_{1}^{*}\left(\beta+\mathrm{r}_{2}\right)\right]}{\beta+\mathrm{r}_{2}}, \mathrm{p}_{38}=\frac{\mathrm{r}_{2}\left[1-\mathrm{g}_{1}^{*}\left(\beta+\mathrm{r}_{2}\right)\right]}{\beta+\mathrm{r}_{2}}, \\
& \mathrm{p}_{40}=\mathrm{g}_{1}^{*}(\beta), \mathrm{p}_{46}=1-\mathrm{g}_{1}^{*}(\beta), \mathrm{p}_{52}=\frac{\beta_{1}}{\beta_{1}+\mathrm{r}_{2}}=\mathrm{p}_{64}=\mathrm{p}_{73}, \\
& \mathrm{p}_{59}=\frac{\mathrm{r}_{2}}{\beta_{1}+\mathrm{r}_{2}}=\mathrm{p}_{69}=\mathrm{p}_{79}, \mathrm{p}_{80}=\mathrm{g}^{*}(\beta), \mathrm{p}_{89}=1-\mathrm{g}^{*}(\beta)
\end{aligned}
$$

It can be verified that

$$
\begin{aligned}
& \mathrm{p}_{01}=\mathrm{p}_{12}+\mathrm{p}_{18}=\mathrm{p}_{23}+\mathrm{p}_{24}+\mathrm{p}_{25}+\mathrm{p}_{28}=\mathrm{p}_{30}+\mathrm{p}_{37}+\mathrm{p}_{38}=\mathrm{p}_{40}+ \\
& \mathrm{p}_{46}=\mathrm{p}_{52}+\mathrm{p}_{59}=\mathrm{p}_{64}+\mathrm{p}_{69}=\mathrm{p}_{73}+\mathrm{p}_{79}=\mathrm{p}_{80}+\mathrm{p}_{89}=\mathrm{p}_{98}=1(3.2)
\end{aligned}
$$


State Transition Diagram

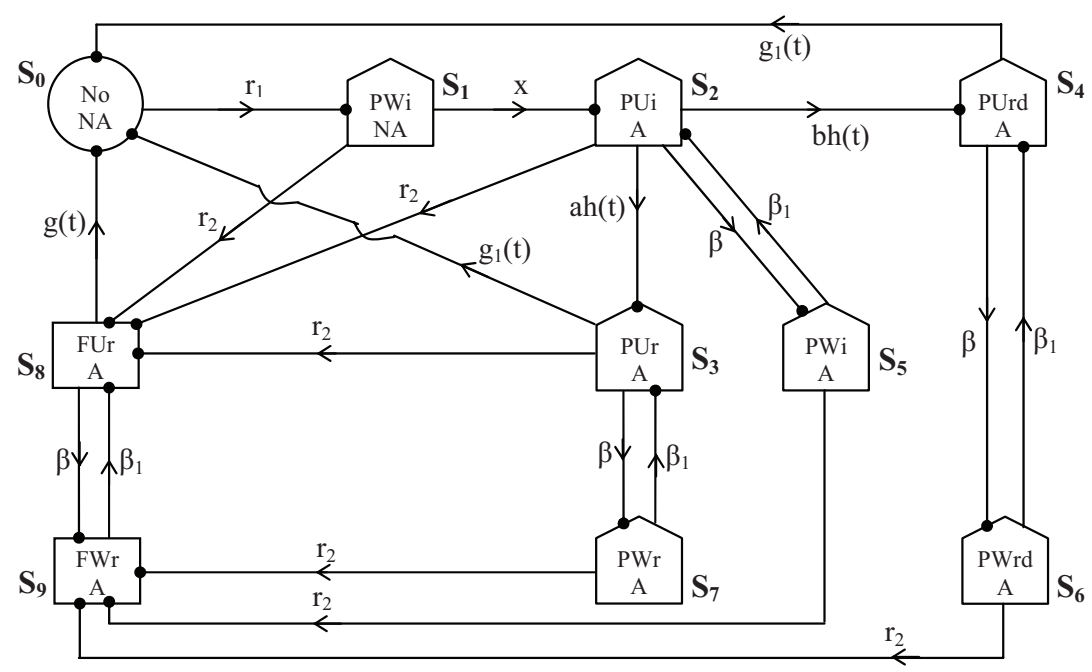

Figure 1. Model
- Transition point
Up-state
Partially failed state
Failed state

The mean sojourn times $\mu_{i}$ in the state $S_{i}$ are

$$
\begin{aligned}
& \mu_{0}=\int_{0}^{\infty} \mathrm{p}(\mathrm{T}>\mathrm{t}) \mathrm{dt}=\frac{1}{\mathrm{r}_{1}}, \mu_{1}=\frac{1}{\mathrm{x}+\mathrm{r}_{2}}, \mu_{2}=\frac{1-\mathrm{h}^{*}\left(\beta+\mathrm{r}_{2}\right)}{\beta+\mathrm{r}_{2}}, \\
& \mu_{3}=\frac{1-\mathrm{g}_{1}^{*}\left(\beta+\mathrm{r}_{2}\right)}{\beta+\mathrm{r}_{2}}, \mu_{4}=\frac{1-\mathrm{g}_{1}^{*}(\beta)}{\beta}, \mu_{5}=\frac{1}{\beta_{1}+\mathrm{r}_{2}}=\mu_{6}=\mu_{7} \\
& \mu_{8}=\frac{1-\mathrm{g}^{*}(\beta)}{\beta}, \mu_{9}=\frac{1}{\beta_{1}}
\end{aligned}
$$

\section{Reliability and Mean Time to System Failure (MTSF)}

Let $\emptyset_{j}(\mathrm{t})$ be the cdf of first passage time from regenerative state i to a failed state. Regarding the failed state as absorbing state, we have the following recursive relations for $\emptyset_{\mathrm{j}}(\mathrm{t})$ :

$$
\varnothing_{\mathrm{i}}(\mathrm{t})=\sum_{\mathrm{i}, \mathrm{j}} \mathrm{Q}_{\mathrm{ij}}(\mathrm{t}) \subseteq \varnothing_{\mathrm{j}}(\mathrm{t})+\sum_{\mathrm{i}, \mathrm{k}} \mathrm{Q}_{\mathrm{ik}}(\mathrm{t})
$$

$j=1 ; 2 ; 3,4,5 ; 0,7 ; 0,6 ; 2 ; 4 ; 3$ for $i=(0-5)$ respectively, $\mathrm{k}=8$ for $\mathrm{i}=(1-3) ; \mathrm{k}=9$ for $\mathrm{i}=(5-7)$ respectively and $\mathrm{Q}_{\mathrm{ik}}(\mathrm{t})=0$ for $\mathrm{i}=(0,4)$.
Taking LST of above relations (4.1) and solving for $\varnothing^{* *}(\mathrm{~s})$, we have

$$
\mathrm{R}^{*}(\mathrm{~s})=\frac{1-\varnothing^{* *}(\mathrm{~s})}{\mathrm{s}}
$$

The reliability of the system model can be obtained by taking inverse LT of (4.2).

The mean time to system failure (MTSF) is given by $\lim _{s \rightarrow 0} R^{*}(s)$. Thus,

where,

$$
\operatorname{MTSF}(T)=\frac{N_{10}}{D_{10}}
$$

$\mathrm{N}_{10}=\left(\mu_{0}+\mu_{1}\right) \mathrm{Z}_{10}+\left(\mu_{2}+\mathrm{p}_{25} \mu_{5}\right) \mathrm{Z}_{11}+\left(\mu_{3}+\mathrm{p}_{37} \mu_{7}\right) \mathrm{Z}_{12}+\left(\mu_{4}\right.$ $\left.+\mathrm{p}_{46} \mu_{6}\right) \mathrm{Z}_{13}$

$\mathrm{D}_{10}=\mathrm{Z}_{10}-\mathrm{p}_{30} \mathrm{Z}_{12}-\mathrm{p}_{40} \mathrm{Z}_{13}$ and $\mathrm{Z}_{10}=\left(1-\mathrm{p}_{25} \mathrm{p}_{52}\right)\left(1-\mathrm{p}_{37} \mathrm{p}_{73}\right)\left(1-\mathrm{p}_{46} \mathrm{p}_{64}\right) ; \quad \mathrm{Z}_{11}=\mathrm{p}_{12}\left(1-\mathrm{p}_{37}\right.$ $\left.\mathrm{p}_{73}\right)\left(1-\mathrm{p}_{46} \mathrm{p}_{64}\right)$

$\mathrm{Z}_{12}=\mathrm{p}_{12} \mathrm{p}_{23}\left(1-\mathrm{p}_{46} \mathrm{p}_{64}\right) ; \quad \mathrm{Z}_{13}=\mathrm{p}_{12} \mathrm{p}_{24}\left(1-\mathrm{p}_{37} \mathrm{p}_{73}\right)$

\section{Steady State Availability}

Let $A_{i}(t)$ be the probability that the system is in up-state at instant ' $\mathrm{t}$ ' given that the system entered regenerative state $i$ at $t=0$. The recursive relations for $A_{j}(t)$ are given as 
$\mathrm{A}_{\mathrm{i}}(\mathrm{t})=\mathrm{M}_{\mathrm{i}}(\mathrm{t})+\sum_{\mathrm{i}, \mathrm{j}} \mathrm{q}_{\mathrm{ij}}(\mathrm{t}) \odot \mathrm{A}_{\mathrm{j}}(\mathrm{t})$

$\mathrm{j}=1 ; 2,8 ; 3,4,5,8 ; 0,7,8 ; 0,6 ; 2,9 ; 4,9 ; 3,9 ; 0,9 ; 8$ for

$\mathrm{i}=(0-8)$ for $\mathrm{i}=(0-8)$ respectively and

$M_{i}(t)=0$ for $i=(4,8,9)$ while $M_{0}(t)=e^{-r_{1} t}, M_{1}(t)=e^{-\left(x+r_{2}\right) t}$,

$M_{2}(t)=e^{-\left(\beta+r_{2}\right) t} \bar{H}(t), M_{3}(t)=e^{-\left(\beta+r_{2}\right) t} \bar{G}_{1}(t)$,

$\mathrm{M}_{5}(\mathrm{t})=\mathrm{e}^{-\left(\beta_{1}+\mathrm{r}_{2}\right) t}=\mathrm{M}_{6}(\mathrm{t})=\mathrm{M}_{7}(\mathrm{t})$

Taking LT of relations (5.1) and solving for $\mathrm{A}_{0}^{*}(\mathrm{~s})$. The steady state availability can be determined as

$$
\begin{gathered}
\mathrm{A}_{0}(\infty)=\lim _{s \rightarrow 0} s \mathrm{~A}_{0}^{*}(\mathrm{~s}) \\
\mathrm{A}_{0}=\frac{\mathrm{N}_{11}}{\mathrm{D}_{11}} \text { where, } \\
\mathrm{N}_{11}=\mathrm{p}_{80}\left[\left(\mathrm{M}_{0}+\mathrm{M}_{1}\right) \mathrm{Z}_{10}+\left(\mathrm{M}_{2}+\mathrm{p}_{25} \mathrm{M}_{5}\right) \mathrm{Z}_{11}+\left(\mathrm{M}_{3}+\mathrm{p}_{37} \mathrm{M}_{7}\right)\right. \\
\left.\mathrm{Z}_{12}-\mathrm{p}_{46} \mathrm{M}_{6} \mathrm{Z}_{13}\right] \\
\mathrm{D}_{11}=\left[\mathrm{p}_{80}\left(\mu_{0}+\mu_{1}\right)+\mu_{8}+\mathrm{p}_{18} \mathrm{p}_{89} \mu_{9}\right] \mathrm{Z}_{10}+\left[\mathrm{p}_{80}\left(\mu_{2}+\mathrm{p}_{25} \mu_{5}\right)+\right. \\
\left.\left(\mathrm{p}_{25} \mathrm{p}_{59}+\mathrm{p}_{28} \mathrm{p}_{89}\right) \mu_{9}\right] \mathrm{Z}_{11}+\left[\mathrm{p}_{80}\left(\mu_{3}+\mathrm{p}_{37} \mu_{7}\right)-\mathrm{p}_{30} \mu_{8}+1\right. \\
\left.\left(\mathrm{p}_{37} \mathrm{p}_{79}+\mathrm{p}_{38}\right) \mu_{9}\right] \mathrm{Z}_{12}+\left[\mathrm{p}_{80}\left(\mu_{4}+\mathrm{p}_{46} \mu_{6}\right)-\mathrm{p}_{40} \mu_{8}\right. \\
\left.+\mathrm{p}_{46} \mathrm{p}_{69} \mu_{9}\right] \mathrm{Z}_{13} \text { and } \\
\mathrm{Z}_{10}, \mathrm{Z}_{11}, \mathrm{Z}_{12}, \mathrm{Z}_{13} \text { are already specified. }
\end{gathered}
$$

\section{Busy Period Analysis}

Let $B_{j}(t)$ be the probability that the server is busy in repairing the unit at an instant ' $\mathrm{t}$ ' given that the system entered regenerative state $i$ at $t=0$. The recursive relations for $B_{j}(t)$ are given as

$$
\mathrm{B}_{\mathrm{i}}(\mathrm{t})=\mathrm{W}_{\mathrm{i}}(\mathrm{t})+\sum_{\mathrm{i}, \mathrm{j}} \mathrm{q}_{\mathrm{ij}}(\mathrm{t}) \odot \mathrm{B}_{\mathrm{j}}(\mathrm{t})
$$

$j=1 ; 2,8 ; 3,4,5,8 ; 0,7,8 ; 0,6 ; 2,9 ; 4,9 ; 3,9 ; 0,9 ; 8$ for $\mathrm{i}=(0-8)$ respectively and

$\mathrm{W}_{\mathrm{i}}(\mathrm{t})=0$ for $\mathrm{i}=(0,1,5-7,9)$ while $\mathrm{W}_{2}(\mathrm{t})=\mathrm{e}^{-(\beta+\mathrm{r} 2) t} \overline{\mathrm{H}}(\mathrm{t})$,

$\mathrm{W}_{3}(\mathrm{t})=\mathrm{e}^{-(\beta+\mathrm{r}) t} \overline{\mathrm{G}}_{1}(\mathrm{t}), \mathrm{W}_{4}(\mathrm{t})=\mathrm{e}^{-\beta \mathrm{t}} \overline{\mathrm{G}}_{1}(\mathrm{t}), \mathrm{W}_{10}(\mathrm{t})=\mathrm{e}^{-\beta \mathrm{t} t} \overline{\mathrm{G}}(\mathrm{t})$

Taking LT of relations (6.1) and solving for $\mathrm{B}_{0}^{*}(\mathrm{~s})$. The busy per iod of the server can be obtained as

$$
\begin{gathered}
\mathrm{B}_{0}(\infty)=\lim _{\mathrm{s} \rightarrow 0} \mathrm{sB}_{0}^{*}(\mathrm{~S}) \\
\mathrm{B}_{0}=\frac{\mathrm{N}_{12}}{\mathrm{D}_{11}} \text {, where, }
\end{gathered}
$$

$\mathrm{N}_{12}=\mathrm{W}_{8} \mathrm{Z}_{10}+\mathrm{P}_{80} \mathrm{~W}_{2} \mathrm{Z}_{11}+\left(\mathrm{P}_{80} \mathrm{~W}_{3}-\mathrm{P}_{30} \mathrm{~W}_{8}\right) \mathrm{Z}_{12}+\left(\mathrm{P}_{80} \mathrm{~W}_{4}-\mathrm{P}_{40}\right.$ $\left.\mathrm{W}_{8}\right) \mathrm{Z}_{13}$ and $\mathrm{D}_{11}, \mathrm{Z}_{10}, \mathrm{Z}_{11}, \mathrm{Z}_{12}, \mathrm{Z}_{13}$ are already specified.

\section{Expected Number of Visits by the Server}

Let $\mathrm{N}_{\mathrm{j}}(\mathrm{t})$ be the expected number of visits by the server in $(0, t]$ given that the system entered the regenerative state $i$ at $\mathrm{t}=0$. The recursive relations for $\mathrm{N}_{\mathrm{j}}(\mathrm{t})$ are given as

$$
\mathrm{N}_{\mathrm{j}}(\mathrm{t})=\sum_{\mathrm{i}, \mathrm{j}} \mathrm{Q}_{\mathrm{ij}}(\mathrm{t}) \subseteq\left[\mathrm{C}+\mathrm{N}_{\mathrm{j}}(\mathrm{t})\right]
$$

$\mathrm{j}=1 ; 2,8 ; 3,4,5,8 ; 0,7,8 ; 0,6 ; 2,9 ; 4,9 ; 3,9 ; 0,9 ; 8$ for $\mathrm{i}=(0-8)$ respectively and

$C=\left\{\begin{array}{l}0 \text { for } i=(0,2-9) \\ 1 \text { for } i=1\end{array}\right.$

Taking LST of relations (7.1) and solving for $\mathrm{N}_{0}^{* * *}(\mathrm{~s})$. The expected number of visits per unit time is given by

$$
\begin{gathered}
\mathrm{N}_{0}(\infty)=\lim _{\mathrm{s} \rightarrow 0} \mathrm{sN}_{0}^{* *}(\mathrm{~s}) \\
\mathrm{N}_{0}=\frac{\mathrm{N}_{13}}{\mathrm{D}_{11}} \text {, where }
\end{gathered}
$$

$\mathrm{N}_{13}=\mathrm{p}_{80} \mathrm{Z}_{10}$ and $\mathrm{D}_{11}, \mathrm{Z}_{10}$ are already specified.

\section{Profit Analysis}

Profit incurred to the system for the model in steady-state can be evaluated as

$$
\mathrm{P}=\mathrm{K}_{0} \mathrm{~A}_{0}-\mathrm{K}_{1} \mathrm{~B}_{0}-\mathrm{K}_{2} \mathrm{~N}_{0} \text {, where }
$$

$\mathrm{K}_{0}=$ Fixed Revenue per unit up time of the system,

$\mathrm{K}_{1}=$ Fixed Cost per unit up time for which server is busy,

$\mathrm{K}_{2}=$ Fixed Cost per unit visit by the server.

\section{Particular Case}

Suppose $g(t)=\alpha e^{-\alpha t}, g_{1}(t)=\alpha_{1} e^{-\alpha_{1} t}, h(t)=\theta e^{-\theta t}$

The transition probabilities $\mathrm{P}_{01}, \mathrm{P}_{12}, \mathrm{P}_{18}, \mathrm{P}_{52}, \mathrm{P}_{59}, \mathrm{P}_{64}, \mathrm{P}_{69}$, $\mathrm{P}_{73}, \mathrm{P}_{79}, \mathrm{P}_{98}$, are same as in (3.1) while

$\mathrm{p}_{23}=\frac{\mathrm{a} \theta}{\beta+\mathrm{r}_{2}+\theta}, \mathrm{p}_{24}=\frac{\mathrm{b} \theta}{\beta+\mathrm{r}_{2}+\theta}, \mathrm{p}_{25}=\frac{\beta}{\beta+\mathrm{r}_{2}+\theta}, \mathrm{p}_{28}=\frac{\mathrm{r}_{2}}{\beta+\mathrm{r}_{2}+\theta}$, $\mathrm{p}_{30}=\frac{\alpha_{1}}{\beta+\mathrm{r}_{2}+\alpha_{1}}, \mathrm{p}_{37}=\frac{\beta}{\beta+\mathrm{r}_{2}+\alpha_{1}}, \mathrm{p}_{38}=\frac{\mathrm{r}_{2}}{\beta+\mathrm{r}_{2}+\alpha_{1}}, \mathrm{p}_{40}=\frac{\alpha_{1}}{\beta+\alpha_{1}}$, $\mathrm{p}_{46}=\frac{\beta}{\beta+\alpha_{1}}, \mathrm{p}_{80}=\frac{\alpha}{\beta+\alpha}, \mathrm{p}_{89}=\frac{\beta}{\beta+\alpha}$

The mean sojourn times $\mu_{j}$ for $i=(0,1,5-7,9)$ in the state $s_{i}$ are same as in (3.3) while

$$
\mu_{2}=\frac{1}{\beta+r_{2}+\theta}, \mu_{3}=\frac{1}{\beta+r_{2}+\alpha_{1}}, \mu_{4}=\frac{1}{\beta+\alpha_{1}}, \mu_{8}=\frac{1}{\beta+\alpha}
$$


also,

$$
\begin{aligned}
& \mathrm{M}_{0}=\frac{1}{\mathrm{r}_{1}}, \mathrm{M}_{1}=\frac{1}{\mathrm{x}+\mathrm{r}_{2}}, \mathrm{M}_{2}=\frac{1}{\beta+\mathrm{r}_{2}+\theta}, \mathrm{M}_{3}=\frac{1}{\beta+\mathrm{r}_{2}+\alpha_{1}}, \\
& \mathrm{M}_{5}=\frac{1}{\beta_{1}+\mathrm{r}_{2}}, \mathrm{M}_{6}=\mathrm{M}_{7} \\
& \quad \text { and } \mathrm{W}_{2}=\frac{1}{\beta+\mathrm{r}_{2}+\theta}, \mathrm{W}_{3}=\frac{1}{\beta+\mathrm{r}_{2}+\alpha_{1}}, \mathrm{~W}_{4}=\frac{1}{\beta+\alpha_{1}}, \\
& \mathrm{~W}_{8}=\frac{1}{\beta+\alpha} \\
& \operatorname{MTFS}(\mathrm{T})=\frac{\mathrm{N}_{10}}{\mathrm{D}_{10}}, \mathrm{~A}_{0}=\frac{\mathrm{N}_{11}}{\mathrm{D}_{11}}, \mathrm{~B}_{0}=\frac{\mathrm{N}_{12}}{\mathrm{D}_{11}}, \mathrm{~N}_{0}=\frac{\mathrm{N}_{13}}{\mathrm{D}_{11}}
\end{aligned}
$$

where

$$
\begin{aligned}
\mathrm{N}_{10}= & \left(\mu_{0}+\mu_{1}\right) \mathrm{Z}_{10}+\left(\mu_{2}+\mathrm{p}_{25} \mu_{5}\right) \mathrm{Z}_{11}+\left(\mu_{3}+\mathrm{p}_{37} \mu_{7}\right) \mathrm{Z}_{12}+ \\
& \left(\mu_{4}+\mathrm{p}_{46} \mu_{6}\right) \mathrm{Z}_{13} \\
\mathrm{~N}_{11}= & \mathrm{p}_{80}\left[\left(\mathrm{M}_{0}+\mathrm{M}_{1}\right) \mathrm{Z}_{10}+\left(\mathrm{M}_{2}+\mathrm{p}_{25} \mathrm{M}_{5}\right) \mathrm{Z}_{11}+\left(\mathrm{M}_{3}+\mathrm{p}_{37} \mathrm{M}_{7}\right)\right. \\
& \left.\mathrm{Z}_{12}-\mathrm{p}_{46} \mathrm{M}_{6} \mathrm{Z}_{13}\right] \\
\mathrm{N}_{12}= & \mathrm{W}_{8} \mathrm{Z}_{10}+\mathrm{p}_{80} \mathrm{~W}_{2} \mathrm{Z}_{11}+\left(\mathrm{p}_{80} \mathrm{~W}_{3}-\mathrm{p}_{30} \mathrm{~W}_{8}\right) \mathrm{Z}_{12}+\left(\mathrm{p}_{80} \mathrm{~W}_{4}-\mathrm{p}_{40}\right. \\
& \left.\mathrm{W}_{8}\right) \mathrm{Z}_{13} \\
\mathrm{~N}_{13}= & \mathrm{p}_{80} \mathrm{Z}_{10} \\
\mathrm{D}_{10}= & \mathrm{Z}_{10}-\mathrm{p}_{30} \mathrm{Z}_{12}-\mathrm{p}_{40} \mathrm{Z}_{13} \\
\mathrm{D}_{11}= & {\left[\mathrm{p}_{80}\left(\mu_{0}+\mu_{1}\right)+\mu_{8}+\mathrm{p}_{18} \mathrm{p}_{89} \mu_{9}\right] \mathrm{Z}_{10}+\left[\mathrm{p}_{80}\left(\mu_{2}+\mathrm{p}_{25} \mu_{5}\right)+\right.} \\
& \left.\left(\mathrm{p}_{25} \mathrm{p}_{59}+\mathrm{p}_{28} \mathrm{p}_{89}\right) \mu_{9}\right] \mathrm{Z}_{11}+\left[\mathrm{p}_{80}\left(\mu_{3}+\mathrm{p}_{37} \mu_{7}\right)-\mathrm{p}_{30} \mu_{8}+\right. \\
& \left.\left(\mathrm{p}_{37} \mathrm{p}_{79}+\mathrm{p}_{38}\right) \mu_{9}\right] \mathrm{Z}_{12}+\left[\mathrm{p}_{80}\left(\mu_{4}+\mathrm{p}_{46} \mu_{6}\right)-\mathrm{p}_{40} \mu_{8}+\mathrm{p}_{46} \mathrm{p}_{69}\right. \\
& \left.\mu_{9}\right] \mathrm{Z}_{13} \\
\mathrm{Z}_{10}= & \left(1-\mathrm{P}_{25} \mathrm{P}_{52}\right)\left(1-\mathrm{P}_{37} \mathrm{P}_{73}\right)\left(1-\mathrm{P}_{46} \mathrm{P}_{64}\right) ; \\
\mathrm{Z}_{11}= & \mathrm{P}_{12}\left(1-\mathrm{P}_{37} \mathrm{P}_{73}\right)\left(1-\mathrm{P}_{46} \mathrm{P}_{64}\right) ; \\
\mathrm{Z}_{12}= & \mathrm{P}_{12} \mathrm{P}_{23}\left(1-\mathrm{P}_{46} \mathrm{P}_{64}\right) ; \\
\mathrm{Z}_{13}= & \mathrm{P}_{12} \mathrm{P}_{24}\left(1-\mathrm{P}_{37} \mathrm{P}_{73}\right)
\end{aligned}
$$

\section{Conclusion}

From Figure 2 it is clear that mean time to system failure (MTSF) decreases as abnormal weather rate $(\beta)$ increases; while it increases with the increase of normal weather rate $\left(\beta_{1}\right)$ and repair rate $\left(\alpha_{1}\right)$ of the partially failed unit with fixed values of other parameters. Figure 3 shows that the profit decreases with the increase of abnormal weather rate and down time of the system while it increases with increase of normal weather rate as well as repair rate for $\mathrm{K}_{0}=5000, \mathrm{~K}_{1}=500$ and $\mathrm{k}_{2}=150$. On the basis of the outcomes for particular case, it is concluded that a system

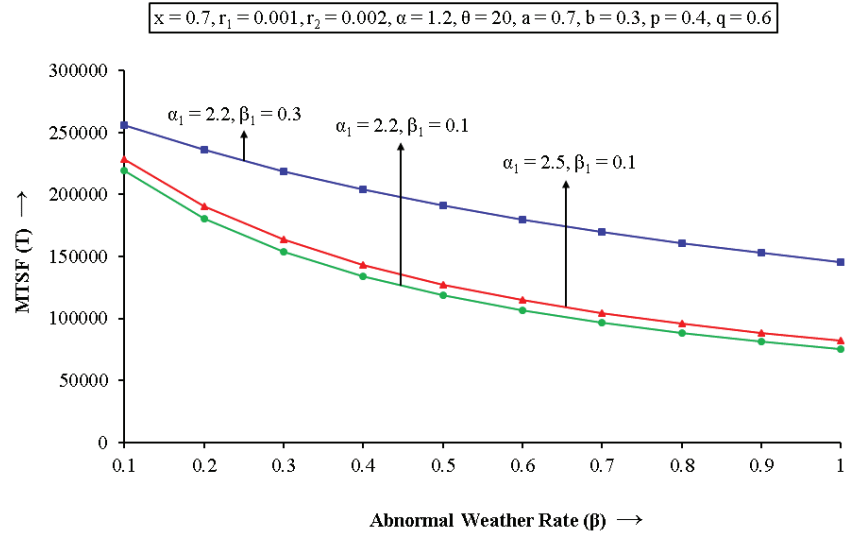

Figure 2. Graph between MTSF v/s Abnormal Weather Rate

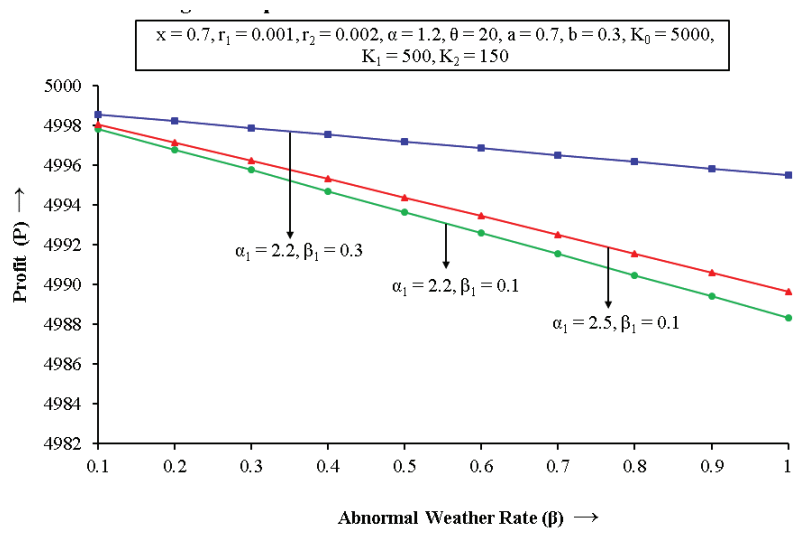

Figure 3. Graph between Profit v/s Abnormal Weather Rate

which works under different weather conditions may be cost-effective at conditional arrival of the server, if

(I) The server attends the system immediately at its complete failure and starts repair without delay.

(II) The server remains with the system in abnormal weather, while the partially failed unit remains operative.

\section{References}

1. Dhillon B S, and Natesan J [1983]. Stochastic analysis of outdoor power system in fluctuating environment, Microelectronics Reliability, vol 23(5), 867-881.

2. Malik S C, and Barak M S [2007]. Probabilistic analysis of a single-server system operating under different weather conditions, Journal of Mathematical Analysis and Approximation Theory, vol 2(2), 173-182. 
3. Pawar D, Malik S C et al. [2010]. Steady state analysis of an operating system with repair at different levels of damages subject to inspection and weather conditions, International Journal of Agricultural and Statistical Sciences, vol 6, No. 1, 225-234.

4. Malik S C, Pawar D et al. [2010]. Analysis of a system working under different weather conditions with on-line repair and random appearance of the server at partial failure stage, International Journal of Statistics and Systems, vol 5, No. 4, 485-496.
5. Malik S C, and Pawar D [2010]. Reliability and economic measures of a system with inspection for on-line repair and no repair activity in abnormal weather, Bulletin of Pure and Applied Sciences, vol 29E(2), 355-368.

6. Pawar D, and Malik S C [2011]. Performance measures of a single unit system subject to different failure modes with operation in abnormal weather, International Journal of Engineering Science and Technology, vol 3(5), 4084-4089. 